\title{
Nonlinear Behavior of Nematic Platelet Dispersions in Shear Flow
}

\author{
M. P. Lettinga, ${ }^{1, *}$ P. Holmqvist, ${ }^{1}$ P. Ballesta, ${ }^{1}$ S. Rogers, ${ }^{1}$ D. Kleshchanok,${ }^{2}$ and B. Struth ${ }^{3}$ \\ ${ }^{1}$ ICS-3, Forschungszentrum Jülich, D-52425 Jülich, Germany \\ ${ }^{2}$ Van t Hoff Laboratory for Physical and Colloid Chemistry, Debye Institute for Nanomaterials Science, \\ Utrecht University, 584CH Utrecht, The Netherlands \\ ${ }^{3}$ DESY, Hasylab, Deutsches Elektronen-Synchrotron, D-22607 Hamburg, Germany
}

(Received 17 July 2012; published 12 December 2012)

\begin{abstract}
Dispersions of platelets in the nematic phase are submitted to large amplitude oscillatory shear flow and probed by high temporal resolution small angle x-ray scattering. The response displays rich dynamic and structural behavior. Under small amplitude deformations we observe an elastic response, while structurally symmetry is broken: a preferential direction of deformation is selected which induces off-plane orientation of the platelets. We associate the elastic responses with the tilting director of the platelets towards the flow direction at all strain amplitudes. At large strain amplitudes there is a yielding transition between elastic and plastic deformation, accompanied by a flipping of the director. At intermediate strain amplitudes the director has a rich dynamic behavior, illustrating the complex motion of platelets in shear flow. These observations are confirmed by steady-shear flow reversal experiments, which underline the unique character of sheared nematic platelet dispersions.
\end{abstract}

DOI: 10.1103/PhysRevLett.109.246001

PACS numbers: 83.85.Hf, 83.80.Xz, 82.70.Dd

Liquid crystals form a unique class of materials due to the combination of highly ordered structure with low mechanical modulus. This is most apparent for dispersions of colloidal rods, where early work of Zocher [1] and Bawden et al. [2] exemplified ordering for very low volume fractions of rods. These observations led Onsager to his seminal thermodynamic description of the relation between crowding and structure [3], used in a variety of fields. The coupling between structure and mechanical properties is rich and nonlinear, although the mechanical modulus of such colloidal dispersions can be very low. There exists a good level of understanding of the interplay between steady shear flow and the different elastic contributions of the nematic structure, as well as the complex response of the director field, which describes the average orientation of the anisotropic particles [4-6]. For this reason sheared nematics are benchmark systems for complex flow studies. Even so, there is a dearth of studies on the viscoelastic character of colloidal liquid crystals. Moreover, the flow behavior of the nematic phase of colloidal platelets is poorly studied, although they are the most ubiquitous colloids in nature and have many practical applications [7].

In this Letter we report the link between the structural and mechanical response of nematic colloidal platelets to large amplitude oscillatory shear flow (LAOS) and steady shear flow reversals. Colloidal platelets, especially clays, often form gels at high concentrations instead of a nematic phase. Although this complicates a fundamental understanding [8], it does result in strong shear-thinning effects due to sheared-induced breakup of these gels [9]. Shear thinning of charge-stabilized systems in the isotropic phase, as studied with in situ small angle x-ray scattering (SAXS), is interpreted in terms of a shear-dependent effective volume fraction due to the aligning platelets [10] that can cause Taylor-Couette instabilities [11]. In situ SANS and birefringence measurements on a columnar phase revealed a complex behavior where platelets orient in different directions [12,13]. The nematic phase of kaolite submitted to LAOS displays a strain hardening and a maximum strain after which the system seems to soften $[14,15]$. The mechanism, however, is unclear.

The combination of LAOS and an appropriate in situ scattering technique is a useful tool to study structural responses underlying complex flow behavior [16-18]. The advantage of LAOS is that material states are probed, which are inaccessible to standard rheological methodologies. In order to elucidate those material states, the responses have to be analyzed in the time domain, where a sequence of physical processes can be identified [17,19]. We apply this approach to Gibbsite particles dispersed in glycerol which form a nematic phase [20], using a highbrilliancy $\mathrm{x}$-ray beam line to ensure sufficient temporal resolution to follow the response of the system to the applied field. We show that the nematic platelets display a simple elastic response at small strain amplitudes, which is accompanied by an anomalous structural response that has the same frequency as the applied field. At sufficiently large strain amplitudes this anomalous structural response disappears, and the system undergoes a transition to elastoplastic behavior where the mechanical response displays a cycle of elasticlike and plasticlike behavior. At all strain amplitudes the elastic response of the system is associated with tilting of the average director of the platelets towards the flow direction. The transition between the different responses is connected with a rich dynamical behavior of the nematic director field. In order to draw direct 
comparisons with previous studies of sheared nematic dispersions $[5,21,22]$, we additionally perform steady-shear flow reversal experiments.

Homemade Gibbsite particles with radius $R=125 \pm$ $16 \mathrm{~nm}$ and thickness $d=11 \pm 4 \mathrm{~nm}$ were dispersed in glycerol at a volume fraction of $12.7 \%$, where this system forms a nematic phase [23]. To monitor the structure in situ we use a recently introduced SAXS setup at beam line P10 (at PETRA III, DESY, Hamburg, Germany) where a vertical deflected beam $(100 \mu \mathrm{m}$ in diameter) was directed through the gradient direction of a plate-plate geometry on an adapted Haake-Mars rheometer [24]. In this geometry, as is indicated in Fig. $1 i i, \varphi(\in[0,2 \pi])$ is the azimuthal angle of the outward normal unit vector $\mathbf{n}$ with the shear flow in the flow-vorticity plane, which is also the plane of the wall, while $\theta(\in[0, \pi])$ is the polar angle with the flowgradient vector. For the dynamic experiments we applied a frequency of $0.04 \mathrm{~Hz}$. Strain amplitudes were reduced from $\gamma_{0}=12.8$ to 0.2 in discrete steps.
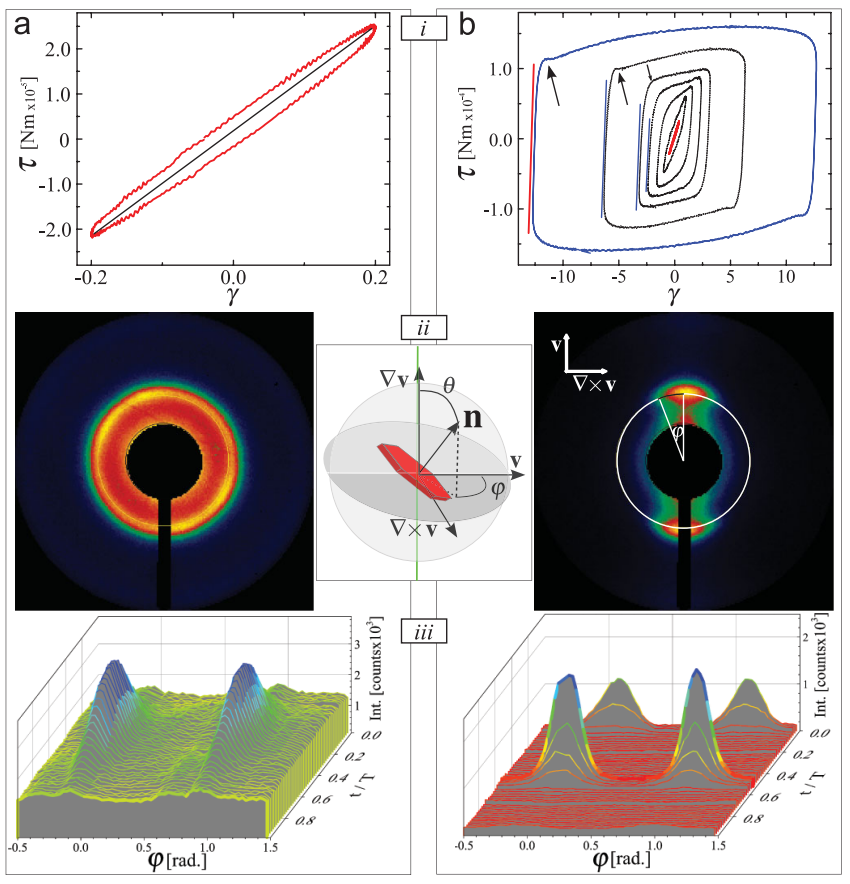

FIG. 1 (color). Mechanical and structural responses for $\gamma_{0}=$ 0.2 (a) and $\gamma_{0}=12.8$ (b). (i) Lissajous representations of the mechanical response to oscillatory shear. A range of responses for smaller $\gamma_{0}$ are also shown in (bi, black curves), showing the development of an overshoot with increasing strain amplitude. The solid lines indicate predominantly elastic parts of the responses. (ii) Scattering patterns as observed during an oscillation. The ring indicates $q_{\max }=0.16 \mathrm{~nm}^{-1}$, where the azimuthal profile is taken, plotted in (iii) for one period of $25 \mathrm{~s}$. The thick lines in iii indicate the time for which the scatter patterns are shown. In the middle a sketch of the scattering geometry, where the green line indicates the $\mathrm{x}$-ray beam and $\mathbf{n}$ the outward normal unit vector.
Lissajous figures where stress, or torque, is plotted vs strain or shear rate are insightful representations of the mechanical response of any material. Hooke's law states that the stress is proportional to the strain for elastic materials. Such elastic behavior is apparent when the Gibbsite dispersion is submitted to small strain oscillations, as shown in Fig. 1(a) $i$, where stress is almost linear with strain. With increasing strain amplitude, an overshoot develops (see arrows in Fig. 1(b)i) where an elastic response (where the stress or torque changes approximately linearly with strain, see lines in Fig. 1(b)i) is identified that is followed sequentially by plastic deformation, indicating that the material is forced to yield. This behavior is typical for a yielding fluid and has been observed for systems ranging from hard [25] and soft spheres [26] to wormlike micelles [17,18,27] and polymers [28]. Furthermore, it is clear from the similarity of the slopes of the elastic portions of the Lissajous curves in Fig. 1(b) $i$ that the elasticity exhibited by the system is nearly constant in magnitude for all large-amplitude responses. A more detailed comparison of these slopes indicates that the elasticity exhibited under LAOS conditions is greater in magnitude than the elasticity of the small amplitude response (inner Lissajous curve in Fig. 1(b)i).

We study the structural origin of the transition from an elastic to an elastoplastic response by time-resolved SAXS. Figure 1ii show snap shots of typical scatter patterns for the smallest and largest strain amplitudes of $\gamma_{0}=0.2$ and $\gamma_{0}=12.8$, respectively. Figure 1iii displays the corresponding azimuthal profiles over one period, taken at the maximum of the structure peaks $q_{\max }$. The small strain amplitude elastic response, Fig. 1(a) $i$, is accompanied by the increase and decrease of the structure peak which takes a full period of oscillation, Fig. 1(a)iii. This is surprising since it is commonly assumed that the overall response of a structure to shear is independent of the direction of deformation, meaning that the structural response has twice the frequency of the applied oscillatory shear field. The large amplitude elastoplastic responses, Fig. 1(b) $i$, are accompanied by short appearances of the structure peak when the material behaves elastically, Fig. 1(b)iii. The elastic response of the system is therefore due to the collective rearrangement of the platelets in the flow field.

To parametrize the structural response, we fit azimuthal profiles as in Fig. 1iii with an orientational distribution function $f(\varphi)=A \exp \left[\alpha P_{2}\left(\cos \varphi-\varphi_{\max }\right)\right]$ (fit not shown). Here $P_{2}$ is the second order Legendre polynomial, $\alpha$ is a measure of the ordering, $\varphi_{\max }$ gives the azimuthal orientation of the nematic director, and $A$ is the peak intensity. From $f(\varphi)$ the orientational order parameter can be calculated: $\left\langle P_{2}(\varphi)\right\rangle=\frac{\int_{0}^{\pi} f(\varphi) P_{2}(\varphi) \sin (\varphi) d \varphi}{\int_{0}^{\pi} f(\varphi) \sin (\varphi) d \varphi}$. As we have no direct access to the polar orientation of the director $\theta_{\max }$ with this set-up, we use the fact that $A$ is proportional to $\theta_{\max }$. To appreciate this proportionality, note that for 
$\theta_{\max }=\pi / 2$ the $\mathrm{x}$-ray beam probes the nematic structure, which is characterized by the face-to-face distance of the platelets. For $\theta_{\max }=0$, when the platelets are oriented with the wall, the center-to-center distance of the platelets is probed. Given the $q$ range of the set-up this means for $\theta_{\max }=0$ that most scattered light falls within the beam stop and $A$ is small, while for $\theta_{\max }=\pi / 2$ the face-to-face scattering is optimal. Thus, as soon as the director rotates from the wall, the face-to-face distance can be probed and $A$ will increase. $\left\langle P_{2}(\varphi)\right\rangle, \varphi_{\max }$, and $A$ are obtained throughout an oscillation for $\gamma_{0}=0.2,2.4$, and 12.8 as is shown in Fig. 2. In the lower panels we display the stress response and the applied strain as functions of the normalized time throughout an oscillation.

The elastic stress response at small strain amplitude is accompanied by a transition from a powder-like scatter pattern at maximum negative strain, where there is a distinct peak at $q_{\max }$ but no peak in the azimuthal profile,
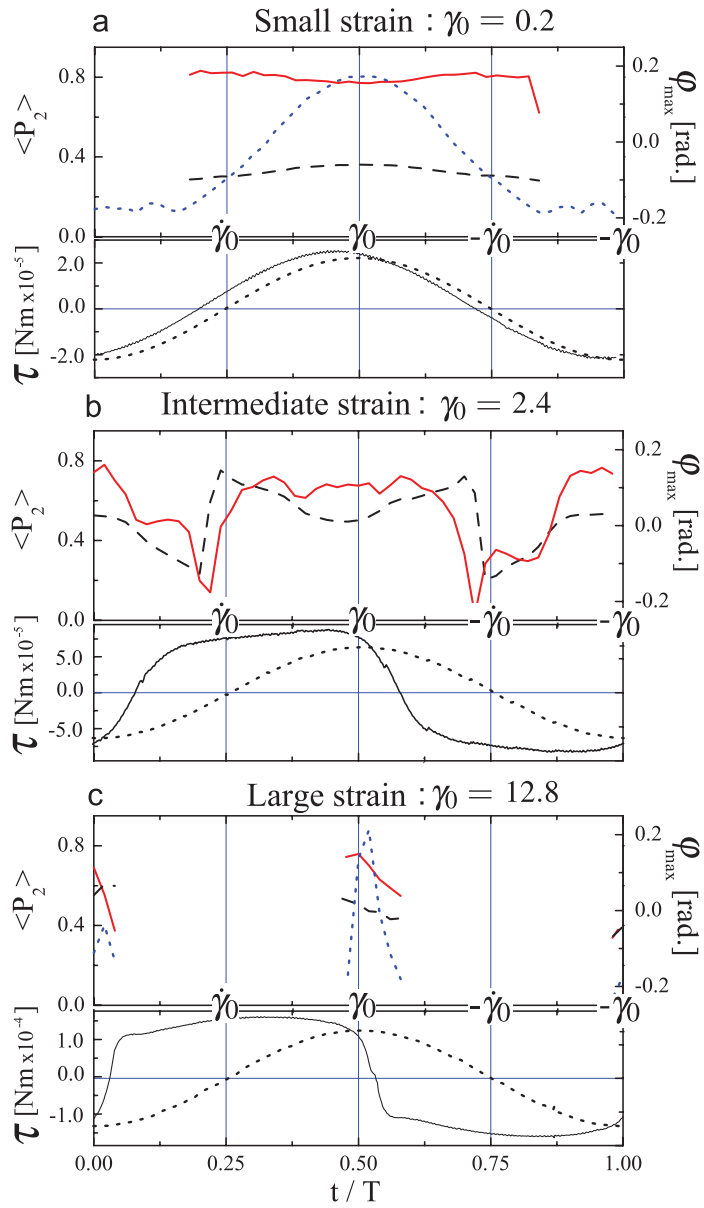

FIG. 2 (color). Response to an oscillatory shear field at various strain amplitudes [33] throughout one period $T$. Top panels: orientational order parameter (solid red line); $\varphi_{\max }$ (dashed black line); peak intensity (dotted blue line, not shown for $\gamma_{0}=2.4$ for clarity). The bottom panels show the applied strain (dotted line) and the torque $\tau$ (solid line). The strain and rate extrema are indicated. to a single peak in the azimuthal profile at $\varphi_{\max } \approx \frac{1}{2} \pi$, at maximum positive strain. As illustrated in Fig. 3(a), the strong structure ring at maximum negative strain at $q_{\max }$ indicates the formation of multi-domains with directors tilted away from the normal of the wall, $\theta_{\max }>0$; the single peak at maximum positive strain corresponds to alignment of the director in the flow direction, while $q_{\max }$ corresponds to a face-to-face distance of the platelets of $37 \mathrm{~nm}$.

For large strain amplitudes there are two sharply defined events, where the director quickly passes through the flow direction, as illustrated in Fig. 3(c). These reorientation events exactly coincide with the elastic portions of the rheological responses (see Fig. 1(b)i) and take place at strain extrema where the flow is reversed. Between the elastic reorientation events there is only scattering at very low $q$, indicating that platelets align with the wall facilitating lamellar flow. In this regime, the material acquires strain in an irreversible, or plastic manner. The boundary between the elastic and plastic responses is marked by a small stress overshoot. The physical interpretation of the elastic-to-plastic transition, the flipping of the director, is contrasted to most other soft matter systems where structural entities (entanglements, cages, etc.) are destroyed. The nematic platelets behave more like lamellar materials such as amphiphilic systems [29] and smectic liquid crystals [24] which display transients caused by the reorientation of the director from the flow to the vorticity direction.
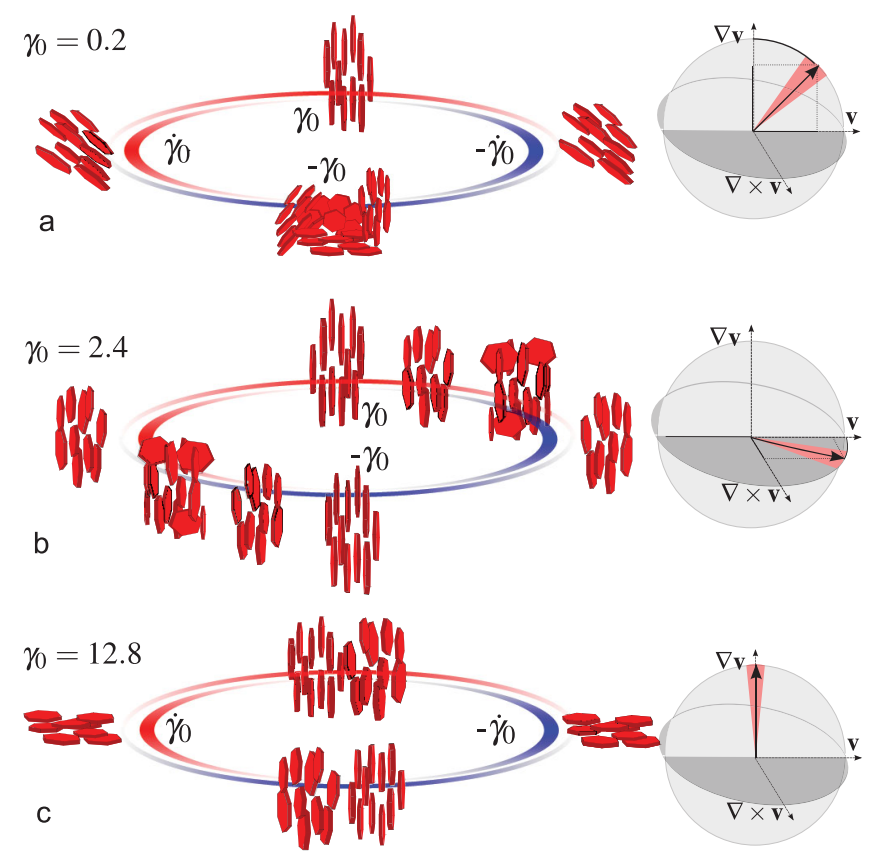

FIG. 3 (color). Cartoon of dynamic behavior during one period for different strain amplitudes. The unit spheres indicate the orientation of the director for the cartoon frames at $-\dot{\gamma}$, while the ordering is sketched by the red cone. 
When deformed by intermediate strain amplitudes the dynamic response is particularly rich. In contrast to the response to large strain amplitudes, intermediate strain amplitudes induce maximum ordering at maximum strain (where the shear rate is instantaneously zero). In contrast to the small strain amplitudes the maximum ordering events are observed at both strain extrema when intermediate strain amplitudes are applied. Thus, there is no selected direction of strain. We identify the following sequence of events, which is illustrated in Fig. 3(b): at maximum strain the director points in the flow direction; with increasing shear rate the domain director tumbles towards the vorticity direction, thus reducing the torque on the platelets, while the orientational distribution widens; at maximum shear rate the director flips, followed by a rotation towards the flow direction. The sequence repeats in the other direction.

We identify a strain bifurcation in the sequence of strain amplitudes that were applied: the system selects a specific direction of deformation which is frozen into its memory as the strain amplitude is reduced below a critical value. Structural responses with the same frequency as the applied deformation are often interpreted as being a signature of a sheared system which has not reached its stationary state. The observed responses are, however, stable over intervals of time on the order of hours and independent of the flow history. Furthermore, the transition from elastic to elastoplastic behavior is independent of the frequency in the range studied $(0.01$ to $0.16 \mathrm{~Hz})$ and also observed when water is used as a solvent.

We can rationalize the transition to a symmetry-broken state by noting that the total strain of 0.4 that could possibly be acquired from $-\gamma_{0}$ to $\gamma_{0}$ in the smallest amplitude case is only enough to tilt the director towards the flow direction $\left(\theta_{\max } \approx \pi / 2\right)$, but no further. When the flow is reversed, the director is pushed back, returning to its initial orientation, as indicated in Fig. 3(a). At intermediate strain amplitude sufficient strain is acquired such that the director is tilted into the flow direction before the maximum strain is achieved. We therefore associate instantaneous elastic deformation with the tilting of the director toward to flow direction at all strain amplitudes. Since the torque on the particles is high when the director lies along the flow direction, relaxation occurs via the tilting of the director into the vorticity direction when the system is strained beyond this point, as we observe for $\gamma_{0}=2.4$ in Fig. 3(b). The sequential widening of the orientational distribution could reflect breaking up of the nematic domain, facilitating the flipping of the director. At large strain amplitudes the critical strain is acquired very shortly after flow reversal and the aforementioned sequence happens abruptly.

Tumbling and widening of the orientational distribution is typical of nematic phases composed of rod-like particles in steady shear flow $[4,5,21,22,30]$ and has also been predicted for nematic platelets $[31,32]$. In order to make a direct comparison with previous studies of sheared nematic dispersions, we perform steady-shear flow reversal experiments, where the sign of the shear flow is changed at $t=0$. This is a standard rheological test to study the tumbling behavior of (colloidal) liquid crystals [5,21,22]. We show in Fig. 4 the responses for $\dot{\gamma}=0.08,0.32$, and $3.2 \mathrm{~s}^{-1}$, plotting $\varphi_{\max }$ and $\left\langle P_{2}(\varphi)\right\rangle$ as a function of the acquired strain after flow reversal at time $t=0$. The stress responses are all overdamped (data not shown), suggesting flow alignment [30]. The response to the lowest shear rate $\left(\dot{\gamma}=0.08 \mathrm{~s}^{-1}\right)$, displays a rich tumbling motion where the director makes full rotations from the flow direction to the vorticity direction and back. The tumbling is accompanied with changes in the order parameter, indicating a widening and narrowing of the orientational distribution function during this tumbling motion. At intermediate shear rate $\left(\dot{\gamma}=0.32 \mathrm{~s}^{-1}\right)$ the distribution widens without rotation of the director. Repeated tumbles are observed at large strains [33]. The same event is observed, but only once, at high shear rate $\left(\dot{\gamma}=3.2 \mathrm{~s}^{-1}\right)$, within a narrow window around $\gamma \approx 10$. This indicates that the director field flips after flow reversal, as is observed for the large strain amplitudes in Fig. 2(c). After the flip the same isotropic structure is observed as under steady shear and quiescent conditions, where anchoring of the nematic director normal to the wall elicits alignment. This complies with an earlier experimental study [12] and suggests strong wall anchoring.

The combination of simultaneous SAXS with LAOS and steady-shear flow reversal we have employed on nematic platelet dispersions has shown the viscoelastic and elastoplastic nature of liquid crystals to be more complex than previously thought. The structural response of the platelets to flow reversal is pronounced and highly complex compared to rodlike systems. Moreover, we observe tumbling in a plane not predicted by theoretical studies [31,32]. LAOS experiments have revealed elastic behavior that is

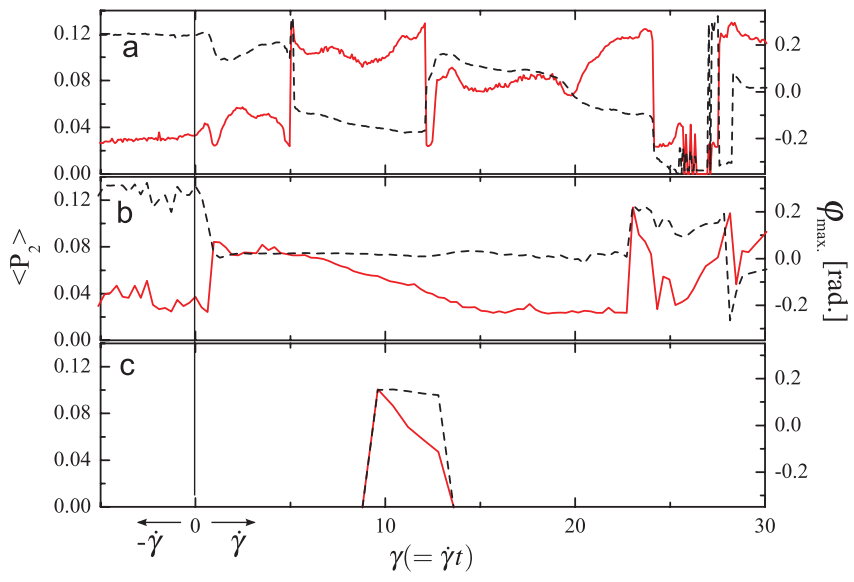

FIG. 4 (color). Response of the orientational order parameter (solid red lines) and the average angle (dashed black lines) to a flow reversal for $\dot{\gamma}=0.08 \mathrm{~s}^{-1}$ (a), $\dot{\gamma}=0.32 \mathrm{~s}^{-1}$ (b), $\dot{\gamma}=3.2 \mathrm{~s}^{-1}$ (c) [33]. 
connected with structural symmetry breaking for small deformations. In response to large deformations we observe a yielding transition between elastic and plastic deformation, accompanied by a flipping of the director. The elastic response of the system is associated at all strain amplitudes with a reorientation of the director into the flow direction, while plastic deformation coincides with the director aligning with the vorticity direction. In order to account for the observed behaviors with theoretical arguments, all possible rotations need to be accounted for, as well as strong anchoring conditions. Moreover, under LAOS conditions, one cannot straightforwardly apply the theory for steady shear flow as special attention needs to be paid to the complex interplay between strain and shear rate effects at each point in the cycle.

This work was performed within the framework of the Transregio SFB TR6, Physics of colloidal dispersions in external fields. The Fundamental Research on Matter (D. K.), the Humboldt Foundation (P. B.), and EU through FP7, project Nanodirect (Grant No. NMP4-SL-2008213948) (S. R.) are thanked for financial support. Rik Wensink is thanked for fruitful discussions. DESY is thanked for access of the beam, and Michael Sprung and Sergej Bondarenko of P10 are acknowledged for setting up the beam line.

*Corresponding author. p.lettinga@fz-juelich.de

[1] H. Zocher, Z. Anorg. Allg. Chem. 147, 91 (1925).

[2] F. C. Bawden, N. W. Pirie, J. D. Bernal, and I. Fankuchen, Nature (London) 138, 1051 (1936).

[3] L. Onsager, Ann. N.Y. Acad. Sci. 51, 627 (1949).

[4] R. Larson, Macromolecules 23, 3983 (1990).

[5] M. P. Lettinga, Z. Dogic, H. Wang, and J. Vermant, Langmuir 21, 8048 (2005)

[6] Y. Tao, W. K. den Otter, and W. J. Briels, Phys. Rev. Lett. 95, 237802 (2005).

[7] G. C. Maitland, Curr. Opin. Colloid Interface Sci. 5, 301 (2000).

[8] L. J. Michot, C. Baravian, I. Bihannic, S. Maddi, C. Moyne, J. F. L. Duval, P. Levitz, and P. Davidson, Langmuir 25, 127 (2009).

[9] A. J. W. ten Brinke, L. Bailey, H. N. W. Lekkerkerker, and G. C. Maitland, Soft Matter 3, 1145 (2007).
[10] I. Bihannic, C. Baravian, J.F. L. Duval, E. Paineau, F. Meneau, P. Levitz, J.P. de Silva, P. Davidson, and L. J. Michot, J. Phys. Chem. B 114, 16347 (2010).

[11] A. M. Philippe, C. Baravian, M. Jenny, F. Meneau, and L. J. Michot, Phys. Rev. Lett. 108, 254501 (2012).

[12] A. B. D. Brown and A. R. Rennie, Phys. Rev. E 62, 851 (2000).

[13] A. B. D. Brown and A. R. Rennie, Chem. Eng. Sci. 56, 2999 (2001).

[14] F. Bossard, T. Aubry, and M. Moan, J. Rheol. 51, 1253 (2007).

[15] M. Moan, T. Aubry, and F. Bossard, J. Rheol. 47, 1493 (2003).

[16] C. R. López-Barrón, L. Porcar, A.P. R. Eberle, and N. J. Wagner, Phys. Rev. Lett. 108, 258301 (2012).

[17] S. A. Rogers, J. Kohlbrecher, and M.P. Lettinga, Soft Matter 8, 7831 (2012).

[18] B. Lonetti, J. Kohlbrecher, L. Willner, J. K. G. Dhont, and M. P. Lettinga, J. Phys. Condens. Matter 20, 404207 (2008).

[19] S. A. Rogers, J. Rheol. 56, 1129 (2012).

[20] F. M. van der Kooij and H. N. W. Lekkerkerker, J. Phys. Chem. B 102, 7829 (1998).

[21] J.-F. Berret, D. C. Roux, G. Porte, and P. Lindner, Europhys. Lett. 32, 137 (1995).

[22] M. Grosso, S. Crescitelli, E. Somma, J. Vermant, P. Moldenaers, and P. L. Maffettone, Phys. Rev. Lett. 90, 098304 (2003).

[23] D. Kleshchanok, J.-M. Meijer, A. V. Petukhov, G. Portale, and H. N. W. Lekkerkerker, Soft Matter 7, 2832 (2011).

[24] B. Struth, K. Hyun, E. Kats, T. Meins, M. Walther, M. Wilhelm, and G. Grübel, Langmuir 27, 2880 (2011).

[25] N. Koumakis, M. Laurati, S. U. Egelhaaf, J. F. Brady, and G. Petekidis, Phys. Rev. Lett. 108, 098303 (2012).

[26] S. A. Rogers, B. M. Erwin, D. Vlassopoulos, and M. Cloitre, J. Rheol. 55, 435 (2011).

[27] J.-F. Berret, Langmuir 13, 2227 (1997).

[28] P. E. Boukany, O. Hemminger, S.-Q. Wang, and L. J. Lee, Phys. Rev. Lett. 105, 027802 (2010).

[29] H. Leist, D. Maring, T. Thurn-Albrecht, and U. Wiesner, J. Chem. Phys. 110, 8225 (1999).

[30] G. Rienäcker and S. Hess, Physica (Amsterdam) 267A, 294 (1999).

[31] A. Singh and A. D. Rey, J. Non-Newtonian Fluid Mech. 94, 87 (2000).

[32] T. Yamamoto, T. Suga, and N. Mori, Phys. Rev. E 72, 021509 (2005).

[33] See Supplemental Material at http://link.aps.org/ supplemental/10.1103/PhysRevLett.109.246001 for movies of all displayed data. 